\title{
Le lait local au Sénégal : intensifier pour développer? Dynamiques socio-techniques et anthropologie des pratiques
}

Thèse de Doctorat (PhD), École doctorale de l'EHESS, Paris, 2016, 392 p.

\section{Sergio Magnani}

\section{OpenEdition}

\section{Journals}

Édition électronique

URL : http://journals.openedition.org/anthropodev/569

DOI : $10.4000 /$ anthropodev.569

ISSN : 2553-1719

\section{Éditeur}

APAD - Association pour l'anthropologie du changement social et du développement

\section{Édition imprimée}

Date de publication : 1 mai 2017

Pagination : 173-175

ISBN : 979-10-93476-04-9

ISSN : 2276-2019

\section{Référence électronique}

Sergio Magnani, «Le lait local au Sénégal : intensifier pour développer? Dynamiques socio-techniques et anthropologie des pratiques », Anthropologie \& développement [En ligne], 45 | 2017, mis en ligne le 01 octobre 2017, consulté le 24 septembre 2020. URL : http://journals.openedition.org/anthropodev/569 ; DOI : https://doi.org/10.4000/anthropodev.569

La revue Anthropologie \& développement est mise à disposition selon les termes de la Licence Creative Commons Attribution 4.0 International. 


\title{
Le lait local au Sénégal : intensifier pour développer ? Dynamiques socio-techniques et anthropologie des pratiques
}

\author{
Sergio Magnani ${ }^{1}$ \\ Thèse de Doctorat (PhD), École doctorale de l'EHESS, Paris, 2016, 392 p.
}

\section{Résumé}

Au Sénégal, les importations de produits laitiers, la volatilité des prix de la poudre de lait, ainsi qu'un décalage important entre croissance de l'offre et de la demande sont autant de facteurs qui creusent le déficit commercial et sont à l'origine d'un regain d'intérêt pour une production locale de lait. Ce renouvellement des enjeux du développement laitier se traduit par une série d'injonctions à l'intensification des systèmes d'élevage. Or, loin d'être neutre, le concept d'intensification a une histoire longue et controversée au Sahel où il a, depuis l'époque coloniale, orienté les principales interventions de développement de l'élevage. Ces dernières, contraignant la mobilité pastorale, privatisant l'accès aux ressources, fragmentant les terres de parcours, ont contribué à accroître la vulnérabilité et le dénuement des populations pastorales.

Cette thèse, fondée sur un travail ethnographique de douze mois au Sénégal et sur l'étude historique des interventions de développement pastoral en milieu semiaride africain et sénégalais, questionne la place centrale qui occupe le paradigme d'intensification. Ce travail propose une ethnographie de la rencontre entre la culture technoscientifique qui légitime l'équation classique "intensification et développement » et les logiques, les pratiques et les modes d'action des éleveurs. L'enquête porte sur trois interventions emblématiques du développement de la production laitière au Sénégal, réalisées dans des terrains contrastés : les milieux périurbains de la capitale Dakar, de Kolda en Haute Casamance et de Richard Toll dans la vallée du fleuve Sénégal.

Plusieurs outils de l'enquête socio-anthropologique de terrain sont mobilisés (120 entretiens avec un large panel d'acteurs, l'observation participante, l'exploration d'archives et de la littérature grise produite localement), dans le contexte d'une recherche menée à plusieurs niveaux. Un premier niveau interroge, sur les terrains choisis, les représentations et les pratiques de certains éleveurs à

${ }^{1}$ E-mail : semagnani@gmail.com

Disponible en ligne : https://tel.archives-ouvertes.fr/tel-01439840 
propos de leurs systèmes d'élevage, de leurs ressources et de leur devenir, des injonctions et des opportunités liées à l'émergence de nouveaux marchés du lait, ainsi que des modèles techniques proposés par les acteurs du développement. Un deuxième niveau concerne les modèles de développement de la production laitière: les structures productives et commerciales de référence, les configurations d'acteurs qui les sous-tendent, les intérêts dont ces acteurs sont porteurs, les systèmes de contraintes dans lesquelles ils se situent, les représentations des pratiques d'élevage et les visions des changements à susciter. Le dernier niveau est celui des actions publiques de développement pastoral au Sénégal, et plus largement au Sahel. Les éléments saillants des deux précédents niveaux sont restitués dans ce contexte pour questionner une idéologie savante et techniciste du développement pastoral, celle de l'intensification des pratiques d'élevage. Les relations entre ces trois niveaux sont explorées à l'aide de trois objets socio-techniques, au cœur des systèmes d'élevage et au centre des injonctions des modèles de développement: l'alimentation du bétail, marqueur des rapports entre systèmes d'élevage et environnement; le lait, objet complexe aux multiples dimensions économiques, sociales et symboliques ; les races bovines, pilier des systèmes d'élevage et révélatrices des évolutions du rapport des éleveurs à la production.

L'analyse montre qu'en concevant l'intensification laitière comme un enjeu et une construction techniques, le cadre d'action publique occulte les contraintes locales de production et commercialisation, la concurrence de la poudre de lait importée et détaxée, et l'absence de politique sectorielle de production et de transformation du lait local. En outre, les résultats suggèrent que la centralité des modèles techniques conduit à légitimer et dépolitiser la soustraction de ressources pastorales induite par les modèles d'aménagement de l'espace à l'œuvre, alors même que de nouvelles dynamiques d'aménagement agricole se profilent. Dans ce contexte, la substitution d'intrants payants aux ressources naturelles est souvent proposée sans aucune considération quant à sa viabilité économique.

La mise en perspective des enquêtes sur les systèmes d'élevage, par le biais des trois objets socio-techniques mentionnés, dessine des pistes de renouveau de l'action publique de développement de l'élevage et de la production laitière. Le rôle irremplaçable des races locales, y compris dans les croisements entrepris localement, invite à reconsidérer la gestion publique de la génétique bovine, en intégrant les visions, les savoirs, les pratiques des éleveurs. Les liens étroits entre pratiques d'alimentation du bétail et politiques d'aménagement de l'espace appellent à la mise en place de politiques territoriales favorisant la préservation ou la construction de complémentarités entre les activités agricoles et pastorales dans des zones écologiquement diversifiées. La dimension marchande du lait local est importante et évolutive mais non exclusive. La pluralité des usages du lait reflète le 
caractère multifonctionnel des élevages, et participe à la reproduction des systèmes. Les résultats suggèrent que la multifonctionnalité des élevages peut contribuer à la durabilité d'un bassin de production laitière, contrairement à ce qui est préconisé par des modèles politiques identifiant la spécialisation productive comme préalable à tout développement du secteur. 